\title{
An electrochemical study of the nickel hydroxide-gold modified electrode employing the surface resistance technique
}

\section{R. Tucceri}

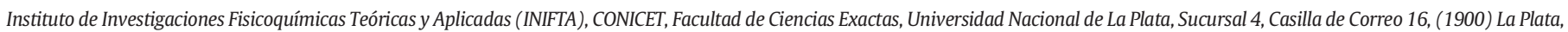
Argentina

\section{A R T I C L E I N F O}

\section{Article history:}

Received 2 December 2015

Received in revised form 25 February 2016

Accepted 6 May 2016

Available online 07 May 2016

\section{Keywords:}

Nickel hydroxide-gold modified electrode

Surface resistance

Nickel hydroxide surface coverage

Nickel adatoms

Field and size effects in thin film electrodes

\begin{abstract}
A B S T R A C T
Simultaneous cyclic voltammetry (CV) and surface resistance (SR) measurements were employed to study the electrochemical behaviour of the nickel hydroxide-gold modified electrode. To this end, firstly, gold film electrodes of different thicknesses were synthesized, and their CV and SR responses within the potential regions corresponding to hydroxide ion adsorption $\left(\mathrm{OH}_{\mathrm{ads}}\right)$ and gold oxide $(\mathrm{AuO})$ formation in alkaline medium were analysed. Then, these gold film electrodes were modified with different nickel hydroxide surface coverages, and their CV and SR responses were compared with those of bare gold films within the same potential regions and electrolyte solution. While cyclic voltammetric responses of the bare gold film electrode and the nickel hydroxide-gold film modified electrode are practically the same within the whole potential range $0.5 \mathrm{~V}<E<0.35 \mathrm{~V}$ (SCE), SR responses are the same only within the potential range $-0.3 \mathrm{~V}<E<0.35 \mathrm{~V}$. Strong differences between the SR responses of the bare gold electrode and the nickel hydroxide-gold modified electrode within the potential range comprised between $-0.5 \mathrm{~V}$ and $-0.3 \mathrm{~V}$ are observed. SR data were interpreted in terms of the field effect and size effect theories formulated to study adsorption processes at thin metallic films. With regard to the bare gold film electrode, the field effect theory allows one to conclude that within the potential region corresponding to hydroxide ion adsorption, $77 \%$ of the electron density charge of gold is involved in the hydroxide ion-gold interaction. Besides, a linear relation between the surface resistance change $(\Delta R)$ and the degree of oxidation $\left(\theta_{\mathrm{ox}}\right)$ at fixed gold film thickness $\left(\phi_{\mathrm{m}}\right)$ is obtained within the potential region corresponding to gold oxide formation. In agreement with the size effect theory, the $\Delta R / \theta_{\mathrm{ox}}$ slope exhibits a linear dependence on $\phi_{\mathrm{m}}^{-2}$. The same SR responses for the nickel hydroxide-gold modified electrode and the bare gold electrode within the potential range $-0.3 \mathrm{~V}<E<0.35 \mathrm{~V}$ indicate that the nickel hydroxide layer does not directly interact with the gold film surface. However, after the reduction of $\mathrm{OH}_{\text {ads }}$ and AuO layers, at potential values more negative than $-0.3 \mathrm{~V}$ (SCE), an increase of the surface resistance is observed for the nickel hydroxide-gold modified electrode, which was attributed to nickel adatoms that directly interact with the gold film surface. On the basis of the electron conduction scattering model employed to describe the interaction of a metal film surface with a foreign atom layer, one can conclude that nickel film growth involves an initial stage in which nickel islands and then, compact structures are formed.
\end{abstract}

(c) 2016 Elsevier B.V. All rights reserved.

\section{Introduction}

Surface resistance (SR) changes of thin metal films have been widely used as a tool to study surface coverage in gas phase [1] as well as in electrochemistry [2]. The resistance of a thin metal film is affected by the presence of lattice defects, foreign entities on its surface and the thickness, which hinder the movement of the electrons in the sample. The SR technique has contributed to the knowledge of the microscopic structure of the metal/solution interface, particularly about the role played by the conduction electrons of the metal in different electrochemical processes [2]. The technique has also been recently extended

E-mail address: rtucce@inifta.unlp.edu.ar. to study the redox process of an electroactive material, such as poly $(0-$ aminophenol), deposited on a gold film electrode [3,4]. In order to extend the use of the SR technique to study different electrochemical systems, in this work we have focused on analysing the effect of nickel hydroxide layers on the electron transport properties of a gold film electrode. Nickel hydroxide is an important electroactive material that, supported on different electrode materials, has been used for many applications of interest [5-8]. In this work, a thin gold film electrode was modified with different nickel hydroxide surface loadings, and the electrode behaviour within potential values corresponding to hydroxide ion adsorption and gold oxide formation was simultaneously studied employing cyclic voltammetry (CV) and SR. While CV allows one to monitor the electroactivity of the nickel hydroxide layer deposited on the gold electrode in terms of ion and electron exchange with the 
solution, complementary SR measurements allows one to study the nickel hydroxide/gold interface in terms of the electron motion change of the gold base substrate due to the presence of the nickel hydroxide layer. To our knowledge, this is the first example of a study on the gold/nickel hydroxide interface employing the SR technique. However, as SR studies of gold film electrodes in alkaline medium are also scarce, we firstly study the SR response of the sole substrate (gold film) in alkaline medium and then, that of the nickel hydroxide-gold modified electrode. Predictions of the field effect and size effect theories formulated for metallic film thicknesses in the order of the mean free path of the conduction electrons [2] are employed to explain the observed SR response. In this work our interest is only to study the effect of nickel species on the conduction properties of the gold film electrode, and then, these species are here considered as "foreign entities" on the gold film surface that impede the electron movement (scatterers) along the film, increasing the resistance of the substrate. Further work is in progress to elucidate the chemical and physical transformations that alter the conducting properties of a gold film after deposition of nickel hydroxide. As the SR technique can be considered as a non-traditional approach in electrochemistry, a brief interpretation of the resistance changes exhibited by a thin metal film in terms of both the surface charge induced by electric fields and the diffuse scattering of conduction electrons from the inside of the metal film to the metal film surface is given to the readers in Section 3.

\section{Experimental}

\subsection{Synthesis of gold films and nickel hydroxide-gold film modified electrodes}

Firstly, five gold film electrodes of different thicknesses $\left(\phi_{\mathrm{m}}=\right.$ $30 \mathrm{~nm} ; 24 \mathrm{~nm} ; 18 \mathrm{~nm} ; 14 \mathrm{~nm}$ and $10 \mathrm{~nm}$ ) were synthesized to study the SR response of the bare gold electrode in the presence of an alkaline solution. Gold films were prepared by vacuum evaporation, as was previously described [2,9-11]. The relationship between the length, $l$, and the width, $w,(G=l / w)$ of these gold film electrodes was 25 . The electrode area was $0.69 \mathrm{~cm}^{2}$. It is well-known that thin metal films obtained by evaporation (low rate of evaporation) exhibit specular surfaces with a relatively low number of defects as compared with a massive metal surface (see Section 3). Each gold film electrode was conditioned electrochemically by cycling the potential between $-0.5 \mathrm{~V}$ and $0.6 \mathrm{~V}$ for 150 cycles at a scan rate of $10 \mathrm{mV} \mathrm{s}^{-1}$ in a deoxygenated $0.2 \mathrm{M}$ $\mathrm{Na}(\mathrm{OH})$ solution. Then, five series of eight nickel hydroxide coated gold film electrodes each were prepared (see columns 3 to 7 in Table 1) to study the CV and SR responses of the nickel hydroxidegold modified electrode. The thickness of the eight gold films of each series is indicated in the heading of columns 3 to 7 in Table 1. The nickel hydroxide surface coverage of each one of the eight films of each series is listed in column 2 of Table 1. It should be indicated that the use of an evaporated gold film as base electrode to deposit nickel hydroxide films leads to reproducible CV and SR data. The good reproducibility can be attributed to the use of a smooth and renewable gold surface, obtained by evaporation, to deposit the nickel hydroxide film in each experiment. Nickel hydroxide was deposited on the gold film electrodes employing the procedure described elsewhere [12], that is, each one of the eight gold films of each series, after being subjected, for a different time period, to a constant potential of $-0.5 \mathrm{~V}$ in a deoxygenated $0.5 \mathrm{M}$ $\mathrm{Ni}\left(\mathrm{NO}_{3}\right)_{2}$ solution, was transferred to a deoxygenated $0.2 \mathrm{M} \mathrm{Na}(\mathrm{OH})$ solution and cycled between $-0.5 \mathrm{~V}$ and $0.6 \mathrm{~V}$ (vs. SCE) at $10 \mathrm{mV} \mathrm{s}^{-1}$ until a stable voltammogram was achieved ( 150 cycles). This procedure allows one to obtain different nickel hydroxide surface coverages $\left(\Gamma_{\mathrm{Ni}(\mathrm{OH}) 2}\right)$ on each one of the eight films of each series. The surface coverage of the different $\mathrm{Au} / \mathrm{Ni}(\mathrm{OH})_{2}$ modified electrodes was estimated from the voltammetric oxidation (or reduction) charge $\left(\mathrm{Q}_{\mathrm{Ni}(\mathrm{OH}) 2}\right)$ evaluated in the supporting electrolyte solution $(0.2 \mathrm{M} \mathrm{NaOH})$ by using the equation $\Gamma_{\mathrm{Ni}(\mathrm{OH}) 2}=Q_{\mathrm{Ni}(\mathrm{OH}) 2} / n F A$, where $Q_{\mathrm{Ni}(\mathrm{OH}) 2}$ is the anodic (or cathodic) voltammetric charge corresponding to the oxidation (or reduction) process, assuming that all the nickel redox sites are electroactive on the voltammetric time scale; $F$ is the Faraday's constant; $A$ is the geometric surface area of the gold electrode; and $n$ is the number of electrons transferred in the $\mathrm{Ni}$ (II) $\Leftrightarrow \mathrm{Ni}$ (III) oxidation (reduction) reaction. As can be seen from column 2 of Table 1 , the sequence of $\Gamma_{\mathrm{Ni}(\mathrm{OH}) 2}$ values was always the same for each one of the five series. A large-area gold grid was used as counter-electrode in all experiments. All the potentials reported in this work are referred to the SCE.

\subsection{Cyclic voltammetry and surface resistance measurements}

Firstly, CV and SR measurements within the potential region comprised between $-0.5 \mathrm{~V}$ and $0.6 \mathrm{~V}$ were performed with each one of the five bare gold film electrodes of different thicknesses indicated above, in contact with a deoxygenated $0.2 \mathrm{M} \mathrm{Na}(\mathrm{OH})$ solution. Then, $\mathrm{CV}$ and SR measurements within the same potential region were carried out with the nickel hydroxide-gold film modified electrodes in contact with the same electrolyte solution. Each one of the nickel hydroxidegold modified electrode listed in Table 1 was successively employed as working electrode in an individual experiment. All measurements were performed employing electrolyte solutions previously deoxygenated by $\mathrm{N}_{2}$ bubbling for $5 \mathrm{~h}$. The reduction of the oxygen content in the electrolyte solution is necessary in order to avoid the interference of the $\mathrm{O}_{2}$ reduction process in the CV and SR responses at potential values more negative than $-0.3 \mathrm{~V}(\mathrm{SCE})$.

The experimental set-up for simultaneous voltammetric and SR measurements on thin film electrodes has previously been described

Table 1

$\Delta R / R$ change of the gold film electrode coated with different nickel hydroxide surface coverage.

\begin{tabular}{|c|c|c|c|c|c|c|}
\hline Goldfilm $^{\mathrm{a}}$ & $\Gamma_{\mathrm{Ni}(\mathrm{OH}) 2} / \mathrm{nmol} \mathrm{cm}^{-2 \mathrm{~b}}$ & $\begin{array}{l}10^{3} \Delta R / R^{\mathrm{c}} \\
(R=20 \Omega) \\
\text { Thickness } \\
\phi_{\mathrm{m}}=30 \mathrm{~nm} \\
\text { (Serie } 1)\end{array}$ & $\begin{array}{l}10^{3} \Delta R / R^{\mathrm{c}} \\
(R=25 \Omega) \\
\text { Thickness } \\
\phi_{\mathrm{m}}=24 \mathrm{~nm} \\
\text { (Serie } 2 \text { ) }\end{array}$ & $\begin{array}{l}10^{3} \Delta R / R^{\mathrm{c}} \\
(R=33 \Omega) \\
\text { Thickness } \\
\phi_{\mathrm{m}}=18 \mathrm{~nm} \\
\text { (Serie 3) }\end{array}$ & $\begin{array}{l}10^{3} \Delta R / R^{\mathrm{c}} \\
(R=43 \Omega) \\
\text { Thickness } \\
\phi_{\mathrm{m}}=14 \mathrm{~nm} \\
(\text { Serie } 4)\end{array}$ & $\begin{array}{l}10^{3} \Delta R / R^{\mathrm{c}} \\
(R=60 \Omega) \\
\text { Thickness } \\
\phi_{\mathrm{m}}=10 \mathrm{~nm} \\
\text { (Serie } 5)\end{array}$ \\
\hline 1 & 0.7 & 1.58 & 1.97 & 2.16 & 3.14 & 5.95 \\
\hline 2 & 1.2 & 2.65 & 3.33 & 4.01 & 5.13 & 9.12 \\
\hline 3 & 1.8 & 3.70 & 4.63 & 5.87 & 7.17 & 13.51 \\
\hline 4 & 3.1 & 6.55 & 8.19 & 10.31 & 12.61 & 23.32 \\
\hline 5 & 6.2 & 7.18 & 8.98 & 10.98 & 13.58 & 23.12 \\
\hline 6 & 9.4 & 7.07 & 8.84 & 11.04 & 13.79 & 23.21 \\
\hline 7 & 16 & 7.27 & 9.10 & 11.11 & 13.79 & 23.19 \\
\hline 8 & 25 & 7.32 & 9.15 & 11.07 & 13.79 & 23.12 \\
\hline
\end{tabular}


in detail [9-11]. The electrochemical cell was also the same as that described previously [9]. The electrode resistance change was measured employing the three-contact method described earlier [10]. The possibility of coupling between the faradaic and measuring currents inside the electrode was taken into account in [10]. It was demonstrated that side effects of the faradaic current passing through the electrode can be neglected when the contacts at the ends of the electrode are symmetrically placed with respect to the central one. Usually, the relative resistance change as a function of potential $(\Delta R / R-E)$ is recorded in SR experiments, where $R$ is the initial resistance value of the gold film and depends on the film thickness. The $R$ value of the eight films of each series is indicated in the heading of columns 3 to 7 of Table 1 .

Voltammetric experiments were performed using a measurement system comprising a PAR model 175 universal programmer, a PAR model 173 potentiostat-galvanostat, and a Philips model 8134 x-y1-y2 recorder. AR grade chemicals were employed throughout. $\mathrm{Ni}\left(\mathrm{NO}_{3}\right)_{2}$ Fluka was employed. $\mathrm{NaOH}$ (Merck) was used without purification. The solutions were prepared with water purified using a Millipore Milli-Q system. The potential drop at the extremes of the film was measured with a Keithley Model 160 voltmeter.

\section{Electronic transport in thin metal films}

The use of the SR changes as an alternative method to study the electrochemical interface was reviewed considering both experimental and theoretical aspects [2]. The most important contributions to electrode resistance changes in electrochemistry are: $(i)$ the field effect, related to the application of an electric field that modifies the electrode conductivity; (ii) the size effect, which refers to conductivity changes brought about by changes of the film thickness; and (iii) adsorption effects.

The field effect precisely refers to the variation of the resistance $\Delta R / R$ with surface charge density $(Q)$, induced by the electric field applied. Interpretation of the field effect on the basis of the free electron model [13] leads to Eq. (1):

$(\Delta \mathrm{R} / R / Q)=\left(n_{0} e \phi_{m}\right)^{-1}$.

In Eq. (1), $n_{\mathrm{o}}$ is the metal electron density, $e$ is the electron charge and $\phi_{\mathrm{m}}$ the film thickness. In the present work we compare the $(\Delta R / R /$ $Q$ ) value obtained within the potential region where hydroxide ion adsorption on gold occurs with the predictions of Eq. (1) related to the field effect.

With regard to size effects, the scattering of conduction electrons at planar interfaces defined by the top and bottom surfaces of a metal film can contribute significantly to the resistivity. In the case of thin metal films, the electrical resistivity, $\rho_{\mathrm{f}}$, is higher than the bulk resistivity, $\rho_{\mathrm{m}}$, of the massive metal of the same structure as the metal film, and the $\rho_{\mathrm{f}} / \rho_{\mathrm{m}}$ ratio decreases with increasing film thickness, $\phi_{\mathrm{m}}$. The theory to account for the size effect was postulated by Fuchs [14] and Sondheimer [15]. The expression for the dependence of the film resistivity, $\rho_{\mathrm{f}}$, as a function of $\phi_{\mathrm{m}}$ can be written as:

$\rho_{f} / \rho_{m}=1+(3 / 8)(1-p) l_{m} / \phi_{m}$

In Eq. (2), $l_{\mathrm{m}}$ is the mean free path of the conduction electrons and $p$ is the specularity parameter $[14,15]$. The specularity parameter represents the probability of an electron being reflected specularly or diffusely at the film surface. The $p$ value ranges from 0 for complete diffuse scattering to 1 for complete specular scattering. At first, it should be considered that thin metal films can be prepared to satisfy the Fuch's model [14] in a sufficient way to exhibit a specularity parameter near 1 (a surface with a smooth mirror-like finish that is free of defects). However, this parameter correlates with the roughness of surface topography and the presence of surface defects. All these imperfections should lead to experimental $p$ values lower than 1 . Our gold films are prepared at low evaporation rates and they show a specularity parameter near 0.91 .

Adsorption on metal film surfaces has also been considered in terms of the electron scattering process. That is, besides the above-mentioned factors, if foreign entities (adsorbates) are present on the film surface, translational symmetry parallel to the interface changes, and additional scattering of the conduction electrons occurs. This electron dispersion effect, brought about by the presence of foreign entities on the metal surface, thereby acting as dispersion centres for the surface reflection of the electrons from the inside of the metal, has been analysed on the basis of Eq. (2). Assuming that the specularity, $p$, decreases with the increase of the surface concentration of foreign scattering centres $\left(\Gamma_{\text {Surf }}\right)$ at the film surface ( $\Delta p=-k_{\mathrm{o}} \Gamma_{\text {Surf }}$ ) and taking into account the relationship between resistance and resistivity, differentiation of Eq. (2) leads to the relationship,

$\Delta R=3 / 8 G\left(\rho_{\mathrm{m}} l_{\mathrm{m}} / \phi_{\mathrm{m}}^{2}\right) k_{0} \Gamma_{\text {Surf }}$

Then, an increase of $\Delta R$ would be expected with the increase in the concentration of foreign entities $\left(\Gamma_{\text {Surf }}\right)$ on the metal film surface. Concerning the mechanism proposed in Eq. (3), $\Gamma_{\text {Surf }}$ has been referred to several interfacial phenomena, electrode surface oxidation, ionic, organic molecular adsorption, interaction of metal surfaces with foreign adatoms during underpotential (UPD) deposition processes, etc. [2]. The nickel hydroxide-gold modified electrode is studied in this work on the basis of Eq. (3), where $\Gamma_{\text {Surf }}$ is associated with the nickel hydroxide surface coverage $\left(\Gamma_{\mathrm{Ni}(\mathrm{OH}) 2}\right)$. As the gold film thickness is also varied, the size effect theory prediction, that is, the linear dependence of the $\Delta R / \Gamma_{\mathrm{Ni}(\mathrm{OH}) 2}$ slope on $\phi_{\mathrm{m}}^{-2}$, according to Eq. (3), is also analysed in this work.

The resistance change within the potential region corresponding to surface oxidation of the gold film electrode is analysed as well. In this case, $\Delta p$ is expressed in terms the degree of oxidation $\left(\theta_{\text {ox }}\right)$. That is, on the assumption that the increase in the surface oxidation of the gold film electrode increases the diffuse scattering of the electrons, one can write $\Delta p=-k \theta_{\text {ox }}$; then, the resistance change $\Delta R$ within the potential region corresponding to gold oxide formation can be expressed as

$\Delta R=3 / 8 \mathrm{G}\left(\rho_{\mathrm{m}} l_{\mathrm{m}} / \phi_{\mathrm{m}}^{2}\right) k \theta_{\mathrm{ox}}$.

As can be seen from Eq. (4), if $k$ is independent of $\theta_{\text {ox }}$, a linear $\Delta R / R$ vs. $\theta_{\text {ox }}$ dependence should be expected. The degree of oxidation of the gold film electrode is defined at each potential $E$ by the ratio $\theta_{\mathrm{ox}}=$ $Q_{\mathrm{ox}} / Q_{\mathrm{T}}$, where $Q_{\mathrm{T}}$ is the voltammetric charge corresponding to the completely oxidised gold film and $Q_{0 x}$ is the oxidation charge at each potential $E$ obtained also from the voltammetric current recorded at $\nu=0.01 \mathrm{~V} \mathrm{~s}^{-1}$.

\section{Results and discussion}

4.1. Voltammetric and surface resistance responses of the gold film electrode in alkaline medium

Simultaneous voltammetric and surface resistance $(\Delta R / R-E)$ responses of a $30 \mathrm{~nm}$ gold film electrode in contact with a deoxygenated $0.2 \mathrm{M} \mathrm{NaOH}$ solution, within the potential range $-0.5 \mathrm{~V}<E<0.6 \mathrm{~V}$, is shown in Fig. 1. With regard to the voltammetric response, it is wellknown that in alkaline solutions, measurable adsorption of $\mathrm{OH}^{-}$on gold starts around $-0.4 \mathrm{~V}$, with increasing surface coverage resulting at more positive potentials [16-18]. The anodic current observed at $E \geq 0.2 \mathrm{~V}$ during the positive potential scan has been attributed to gold oxide formation. In the negative potential scan, the gold oxide is reduced at a potential value around $0.1 \mathrm{~V}$.

The surface resistance has been employed to study adsorption on gold film electrodes in acid and neutral media [2]. However, to our 


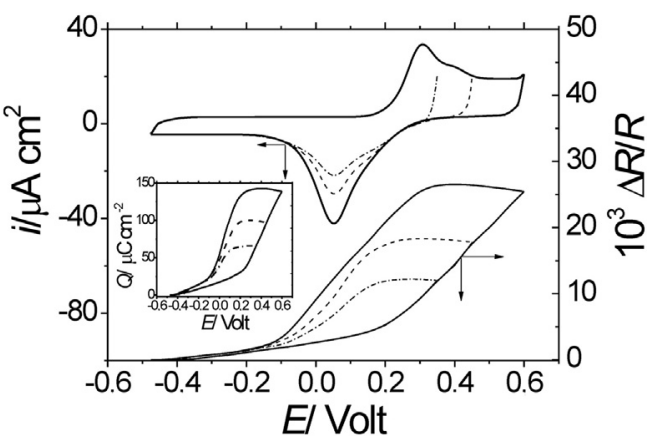

Fig. 1. Voltammetric $(I-E)$ and relative surface resistance $(\Delta R / R-E)$ responses of a $30 \mathrm{~nm}$ gold film electrode in contact with a $0.2 \mathrm{M} \mathrm{Na}(\mathrm{OH})$ solution. Inset: Charge vs. potential $(Q-$ $E$ ) dependence for the same gold film electrode obtained by integration of the $I-E$ response. Potential scan rate: $0.010 \mathrm{~V} \mathrm{~s}^{-1}$.

knowledge, there are few studies related to adsorption on gold film electrodes in alkaline media employing the SR technique [19]. As can be seen by comparing $I-E$ and $\Delta R / R-E$ responses shown in Fig. 1, ions adsorbing on the surface of the gold film electrode cause the increase of the surface resistance in going towards the positive potential scan direction from a potential value about $E=-0.5 \mathrm{~V}$. SR measurements at potential values slightly more negative than $-0.6 \mathrm{~V}$ are not reproducible because the gold film is slowly detached (peeled off) from the base surface, possibly due to incipient hydrogen evolution (bubbles on the gold film surface are visible). The abrupt change of slope in the $\Delta R / R-E$ dependence at about $0.2 \mathrm{~V}$ in going towards the positive potential scan direction shows the beginning of gold oxide formation. The inset in Fig. 1 shows the charge vs. potential ( $Q-E)$ dependence. The $Q-E$ dependence was obtained from the integration of the $I-E$ response shown in Fig. 1. While the SR response depends on the thickness of the gold film electrode (Fig. 2), the voltammetric response and the Q$E$ dependence are independent of this variable (Fig. 1). The inset in Fig. 2 shows $\Delta R / R-E$ responses for the different gold film thicknesses only within the potential region corresponding to hydroxide ion adsorption $(-0.5 \mathrm{~V}<E<0.2 \mathrm{~V})$. Fig. 3 shows the $\Delta R / R-Q$ dependence for the different film thicknesses within the potential region $-0.5 \mathrm{~V}<E<0.2 \mathrm{~V}$. As can be seen from Fig. 3, linear $\Delta R / R-Q$ dependences are obtained for each film thickness. The inset in Fig. 3 shows the comparison of the $(\Delta R / R) / Q$ slopes extracted from Fig. 3 as a function of $\phi_{\mathrm{m}}^{-1}$ with the $(\Delta R / R) / Q$ vs. $\phi_{\mathrm{m}}^{-1}$ dependence predicted by the field effect theory according to Eq. (1). A lower $(\Delta R / R Q) / \phi_{\mathrm{m}}^{-1}$ slope value is predicted by Eq. (1) as compared with the value obtained in the presence of hydroxide ion adsorption. It is well-known that the field effect in electrochemistry is often masked by ionic adsorption in the double-layer

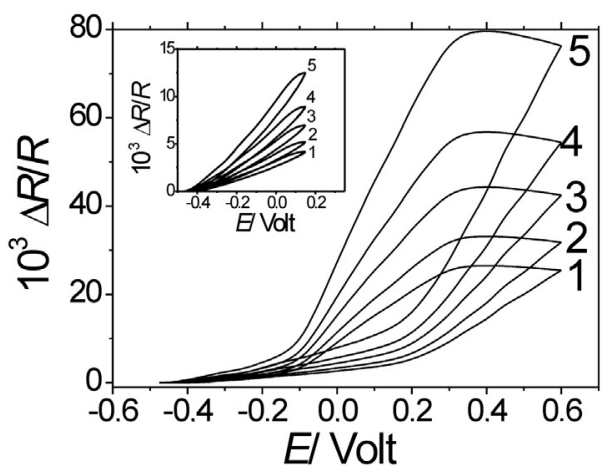

Fig. 2. Relative surface resistance $(\Delta R / R-E)$ responses for gold film electrodes of different thicknesses within the potential region $-0.5 \mathrm{~V}<E<0.6 \mathrm{~V}$. Inset: Relative surface resistance $(\Delta R / R-E)$ responses for the same gold film electrodes within the potential region $-0.5 \mathrm{~V}<E<0.2 \mathrm{~V}$. Thicknesses, 1 : $\varnothing_{\mathrm{m}}=30 \mathrm{~nm} ; 2: \varnothing_{\mathrm{m}}=24 \mathrm{~nm} ; 3$ : $\varnothing_{\mathrm{m}}=18 \mathrm{~nm} ; 4: \varnothing_{\mathrm{m}}=14 \mathrm{~nm} ; 5: \varnothing_{\mathrm{m}}=10 \mathrm{~nm}$. Electrolyte: a $0.2 \mathrm{M} \mathrm{Na}(\mathrm{OH})$ solution. Potential scan rate: $0.010 \mathrm{~V} \mathrm{~s}^{-1}$.

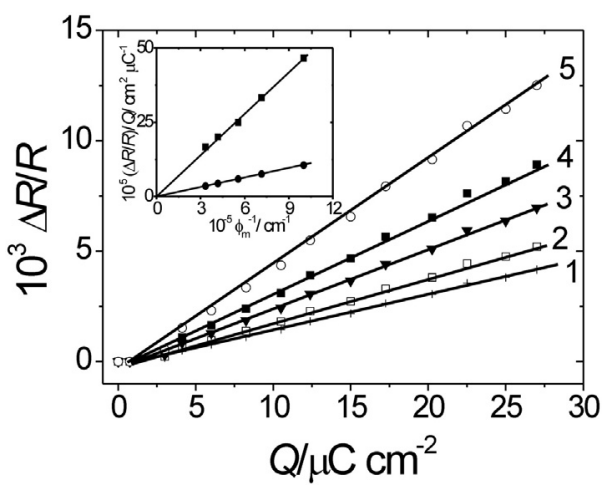

Fig. 3. $(\Delta R / R-Q)$ dependences for the same films indicated in Fig. 2 within the potential region $-0.5 \mathrm{~V}<E<0.2 \mathrm{~V}$. Electrolyte: a $0.2 \mathrm{M} \mathrm{Na}(\mathrm{OH})$ solution. Potential scan rate: $0.010 \mathrm{~V} \mathrm{~s}^{-1}$. Inset: $(\mathbf{\square})(\Delta R / R / Q)$ vs. $\varnothing_{\mathrm{m}}^{-1}$ dependence within the potential region $-0.5 \mathrm{~V}<E<0.2 \mathrm{~V} . \Delta R / R / Q$ values were extracted from the $(\Delta R / R-Q)$ dependences shown in Fig. 3. $(\bullet)(\Delta R / R / Q)$ vs. $\varnothing_{\mathrm{m}}^{-1}$ dependence predicted by the field effect theory.

region [2]. However, it is interesting to note that the comparison of the $(\Delta R / R Q) / \phi_{\mathrm{m}}^{-1}$ slope value, within the potential region $-0.5<E<0.2 \mathrm{~V}$ obtained in the presence of hydroxide ions, with the value extracted from the field effect theory allows one to obtain the $\left(n_{\mathrm{o}} e\right)^{\mathrm{OH}} /\left(n_{\mathrm{o}} e\right)^{\mathrm{FE}}$ ratio. As $n_{\mathrm{o}}$ (electrons/ $\mathrm{cm}^{3}$ ) is the free electron density for gold and $e\left(=1.6 \times 10^{-19}\right.$ coulomb $)$ is the electron charge, $\left(n_{0} e\right)^{\mathrm{OH}} /\left(n_{0} e\right)^{\mathrm{FE}}$ should represent the relationship between the free electron charge density of the electrode in the presence and in the absence of adsorbed hydroxide ions. The $\left(n_{0} e\right)^{\mathrm{OH}} /\left(n_{0} e\right)^{\mathrm{FE}}$ value obtained from data shown in the inset of Fig. 3 is 0.23 . This value should indicate that in the presence of hydroxide ions, $77 \%$ of the electron density charge of gold is involved in hydroxide ion adsorption. This finding seems to be consistent with the hydroxide ion-gold interaction process conceived in terms of partial charge transfers $[17,18]$.

Fig. 4 shows the representation of $\Delta R-\theta_{\mathrm{ox}}$ dependence within the potential region corresponding to gold oxide formation $(0.2 \mathrm{~V}<E<0.6 \mathrm{~V})$ for different gold film thicknesses. The $\Delta R$ was extracted from $\Delta R / R$ measurements by multiplying by the corresponding initial $R$ value for each film thickness (see Table 1 ). The $\Delta R$ value at $E=0.2 \mathrm{~V}$ was taken as reference to assess the resistance change within the potential region corresponding to gold oxide formation for each film thickness. The degree of oxidation, $\theta_{\mathrm{ox}}$, was calculated as indicated in Section 3. As predicted by Eq. (4), a linear $\Delta R-\theta_{\text {ox }}$ dependence is obtained. Also, according to the size effect theory (Eq. (4)), the $\Delta R / \theta_{\text {ox }}$ ratio exhibits a linear dependence on $\phi_{\mathrm{m}}^{-2}$ (inset in Fig. 4) with a $k$ value of about $6.7 \times 10^{-4}$. Linear dependences such as those shown in Fig. 4 are also obtained for the gold oxide reduction process. Then, the SR behaviour of gold film electrodes in alkaline medium seems to respond to field

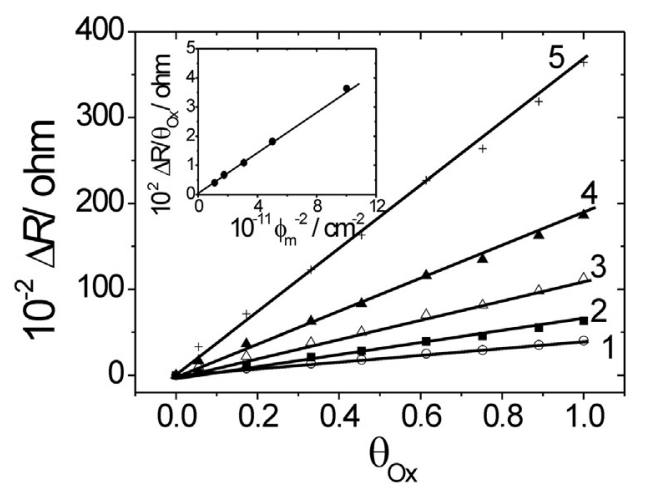

Fig. 4. Surface resistance change as a function of the degree of oxidation ( $\Delta R$ vs. $\left.\theta_{o x}\right)$ for different gold film thicknesses within the potential region $0.2 \mathrm{~V}<E<0.6 \mathrm{~V}$. The same film thicknesses indicated in Fig. 2. Inset: $\Delta R / \theta_{o x}$ vs. $\varnothing_{\mathrm{m}}^{-2}$ dependence. $\Delta R / \theta_{o x}$ values were extracted from the $\Delta R$ vs. $\theta_{o x}$ dependence shown in Fig. 4 . Electrolyte: a $0.2 \mathrm{M}$ $\mathrm{Na}(\mathrm{OH})$ solution. Potential scan rate: $0.010 \mathrm{~V} \mathrm{~s}^{-1}$. 
and size effect theories proposed to interpret conductivity changes in thin metal films due to charge and thickness variations, respectively.

4.2. Voltammetric and surface resistance responses of the nickel hydroxidegold film modified electrodes

The stabilized voltammetric response of a gold film modified with different nickel hydroxide surface coverages $\left(\Gamma_{\mathrm{Ni}(\mathrm{OH}) 2}\right)$ is shown in Fig. 5. This voltammetric response is the weighted sum of the activities of gold and nickel hydroxide. Two redox transitions are observed: a1/c1, which are related to gold-oxide formation and reformation of $\mathrm{Au}^{\circ}$ and $\mathrm{a} 2 / \mathrm{c} 2$, which is due to the $\mathrm{Ni}(\mathrm{II}) / \mathrm{Ni}(\mathrm{III})$ system [20-22]. The shape of the $\mathrm{Ni}(\mathrm{II}) / \mathrm{Ni}(\mathrm{III})$ transition (i.e. peak separation, peak current ratios and peak potentials) is influenced by the $\Gamma_{\mathrm{Ni}(\mathrm{OH}) 2}$ value. The good electronic and permeation properties of the nickel hydroxide films are evidenced by the currents and peak potentials related to a1/c1 processes, which are virtually identical to those observed for the bare gold electrode (Fig. 1). Although the coupling between faradaic and measuring currents is taken into account in SR measurements [2], the high faradaic current passing through the resistive gold electrode within the potential region corresponding to the $\mathrm{Ni}$ (II) $\Leftrightarrow \mathrm{Ni}$ (III) redox process, particularly for $\Gamma_{\mathrm{Ni}(\mathrm{OH}) 2}>10 \mathrm{nmol} \mathrm{cm}^{-2}$ values (inset in Fig. 5), causes some side effects, which are difficult to minimize during SR measurements. Then, simultaneous CV and SR measurements of nickel hydroxide-gold modified electrodes were only performed within the potential range $-0.5 \mathrm{~V}<E<0.35 \mathrm{~V}$. Fig. 6 compares simultaneous $I-E$ and $\Delta R / R-E$ responses for the $30 \mathrm{~nm}$ bare gold film electrode with those of the same film, modified by different nickel hydroxide surface loadings. The $\Gamma_{\mathrm{Ni}(\mathrm{OH}) 2}$ values are the same as those indicated in Fig. 5. Although stabilized voltammetric and surface resistance responses obtained after prolonged potential cycling (around 150 potential cycles at $10 \mathrm{mV} \mathrm{s}^{-1}$ ) are shown in Figs. 5 and 6 , it is well-known that during continuous electrochemical cycling in alkaline solutions the $\mathrm{Au} / \mathrm{Ni}$ electrode undergoes profound physical and/or structural modifications [12]. In this regard, we observe that both voltammetric and SR responses drastically change with the number of potential cycles. As has been reported $[12,22,23]$, nickel hydroxide can exist at least in two different crystallographic forms designed as $\alpha-\mathrm{Ni}(\mathrm{OH})_{2}$ and $\beta-\mathrm{Ni}(\mathrm{OH})_{2}$. The $\alpha-\mathrm{Ni}(\mathrm{OH})_{2}$ is known to be unstable in alkaline medium and converts slowly and irreversibly to $\beta-\mathrm{Ni}(\mathrm{OH})_{2}$. In addition, the oxidation of nickel hydroxide gives another two varieties of oxyhydroxides, $\beta$ and $\gamma$. Thus, the surface concentration of nickel oxyhydroxide increases, and also the gold film electrode undergoes surface reconstruction during multiple potential cycling. The SR technique seems to be a powerful tool to analyse the changes of the electrochemical response on the $\mathrm{Au} / \mathrm{Ni}$ electrode with potential cycling, potential range and electrolyte composition. In this regard, we have previously employed the technique to study the surface

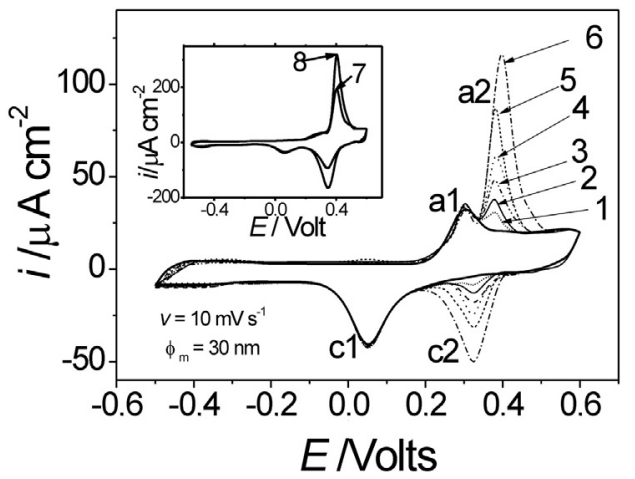

Fig. 5. Voltammetric response of a $30 \mathrm{~nm}$ gold film electrode modified with different nickel hydroxide surface coverages: $\Gamma_{\mathrm{Ni}(\mathrm{OH}) 2}=0.7 \mathrm{nmol} \mathrm{cm}^{-2}(1) ; 1.2 \mathrm{nmol} \mathrm{cm}^{-2}(2)$; $1.8 \mathrm{nmol} \mathrm{cm}{ }^{-2}$ (3); $3.1 \mathrm{nmol} \mathrm{cm}{ }^{-2}$ (4); $6.2 \mathrm{nmol} \mathrm{cm}{ }^{-2}$ (5); $9.4 \mathrm{nmol} \mathrm{cm}{ }^{-2}$ (6). Inset: $\Gamma_{\mathrm{Ni}(\mathrm{OH}) 2}=16 \mathrm{nmol} \mathrm{cm}{ }^{-2}(7) ; 25 \mathrm{nmol} \mathrm{cm}^{-2}(8),(-)$ the bare gold film electrode. Electrolyte: a $0.2 \mathrm{M} \mathrm{Na}(\mathrm{OH})$ solution. Potential scan rate: $0.010 \mathrm{~V} \mathrm{~s}^{-1}$.

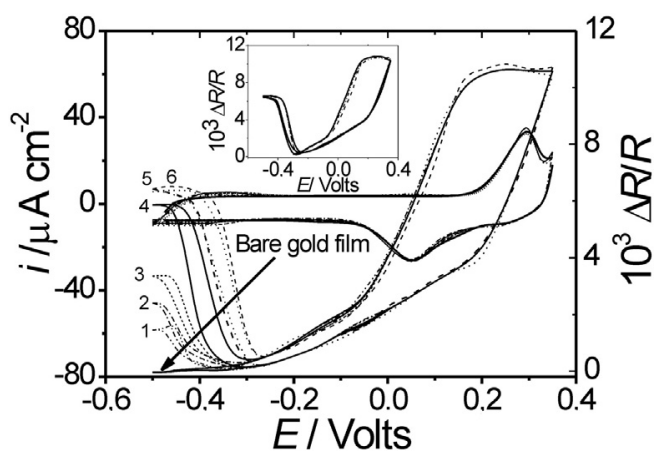

Fig. 6. Voltammetric and $(\Delta R / R-E)$ responses of a $30 \mathrm{~nm}$ gold film electrode modified with different nickel hydroxide coverages: $\Gamma_{\mathrm{Ni}(\mathrm{OH}) 2}=0.7 \mathrm{nmol} \mathrm{cm}^{-2}(1) ; 1.2 \mathrm{nmol} \mathrm{cm}{ }^{-2}$ (2); $1.8 \mathrm{nmol} \mathrm{cm}{ }^{-2}$ (3); $3.1 \mathrm{nmol} \mathrm{cm}{ }^{-2}$ (4); $6.2 \mathrm{nmol} \mathrm{cm}{ }^{-2}$ (5); $9.4 \mathrm{nmol} \mathrm{cm}{ }^{-2}$ (6). (-) The bare gold film electrode. Inset: $(\Delta R / R-E)$ responses for the same gold film electrode modified with nickel hydroxide coverages: $\Gamma_{\mathrm{Ni}(\mathrm{OH}) 2}=9.4 \mathrm{nmol} \mathrm{cm}^{-2}(-) ; 16 \mathrm{nmol} \mathrm{cm}^{-2}$ $(-\cdot-\cdot-) ; 25 \mathrm{nmol} \mathrm{cm}^{-2}(---)$ Electrolyte: a $0.2 \mathrm{M} \mathrm{Na}(\mathrm{OH})$ solution. Potential scan rate: $0.010 \mathrm{~V} \mathrm{~s}^{-1}$.

structural change of silver film electrodes [24]. More work is in progress in our laboratory to study surface structural and chemical composition changes of the $\mathrm{Au} / \mathrm{Ni}$ electrode on the basis of the evolution of the SR response during electrochemical cycling. However, as was indicated, in the present work only stabilized voltammetric and SR responses of the $\mathrm{Au} / \mathrm{Ni}$ electrode are analysed.

Fig. 6 shows that while $I-E$ responses for the different nickel hydroxide-gold modified electrodes and the bare gold electrode are practically the same within the whole potential range, the $\Delta R / R-E$ responses only within the potential region comprised between $-0.3 \mathrm{~V}$ and $0.35 \mathrm{~V}$ are the same. In this regard, a $\Delta R / R$ increase within the potential range comprised between $-0.3 \mathrm{~V}$ and $-0.5 \mathrm{~V}$ in going towards the negative potential direction is observed for the nickel hydroxidegold modified electrodes. This behaviour contrasts with the relatively constant $\Delta R / R$ value for the bare gold film electrode within the same potential region. It should be indicated that to record the $\Delta R / R-E$ change in the presence of nickel hydroxide films, we take as reference the $\Delta R / R$ value at $E=-0.5 \mathrm{~V}$ for the bare gold film electrode (absence of nickel hydroxide surface coverage), which is considered as zero after compensating for the initial resistance $R$ of the gold film electrode. The increase of $\Delta R / R$ in going towards the negative potential scan direction from $-0.3 \mathrm{~V}$ to $-0.5 \mathrm{~V}$ depends on the nickel hydroxide surface coverage value $\left(\Gamma_{\mathrm{Ni}(\mathrm{OH}) 2}\right)$. That is, the higher the $\Gamma_{\mathrm{Ni}(\mathrm{OH}) 2}$ value is, the higher the surface resistance change becomes. However, for $\Gamma_{\mathrm{Ni}(\mathrm{OH}) 2}$ values higher than $3 \mathrm{nmol} \mathrm{cm}{ }^{-2}$, a saturation in the $\Delta R / R$ change is observed (see inset in Fig. 6). Although the $\Delta R / R$ change is magnified as the gold film thickness decreases, saturation in the $\Delta R / R$ change with the $\Gamma_{\mathrm{Ni}(\mathrm{OH}) 2}$ increase is observed for all gold film thicknesses. This effect can be seen clearly in Fig. 7, where $\Delta R$ vs. $\Gamma_{\mathrm{Ni}(\mathrm{OH}) 2}$ dependences for the different

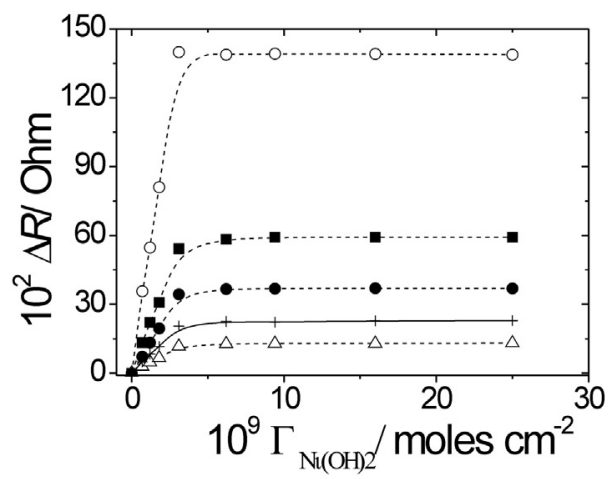

Fig. 7. $\Delta R$ vs. $\Gamma_{\mathrm{Ni}(\mathrm{OH}) 2}$ for gold films of different thicknesses $\left(\varnothing_{\mathrm{m}}\right)$ : (O) $10 \mathrm{~nm}$; (ם) $14 \mathrm{~nm}$; (•) $18 \mathrm{~nm} ;(+) 24 \mathrm{~nm} ;(\Delta) 30 \mathrm{~nm}$. 
gold film thicknesses are plotted together. $\Delta R$ changes were obtained from experimental $\Delta R / R$ changes, employing the corresponding $R$ value for each film thickness (see Table 1). It is also observed in Fig. 7 that for all film thicknesses, $\Delta R$ increases almost linearly within the hydroxide nickel surface coverage range $0<\Gamma_{\mathrm{Ni}(\mathrm{OH}) 2}<3 \mathrm{nmol} \mathrm{cm}^{-2}$. This fact is more clearly seen in Fig. 8. Employing experimental $\Delta R / \Gamma_{\mathrm{Ni}(\mathrm{OH}) 2}$ slopes showed in Fig. 7 and the following parameter values in Eq. (3), $G=25 ; \rho_{\mathrm{m}}=2.4 \times 10^{-6} \Omega \mathrm{cm} ; l_{\mathrm{m}}=22 \mathrm{~nm}$, and the corresponding film thickness, $\phi_{\mathrm{m}}$, a value of $k_{\mathrm{o}}=7.28 \times 10^{6} \mathrm{~cm}^{2} \mathrm{~mol}^{-1}$, independent of $\phi_{\mathrm{m}}$, is obtained. As predicted by the size effect theory for thin metal films [2], the $\Delta R / \Gamma_{\mathrm{Ni}(\mathrm{OH}) 2}$ slope within the surface coverage range $0<\Gamma_{\mathrm{Ni}(\mathrm{OH}) 2}<3 \mathrm{nmol} \mathrm{cm}^{-2}$ follows a linear dependence on $\phi_{\mathrm{m}}^{-2}$ (inset in Fig. 8). Thus, the resistance response of the nickel hydroxide-gold film modified electrode also complies with the size effect theory predictions.

The electrochemical treatment employed in our work (repetitive potential cycling in alkaline solutions) to stabilize the nickel hydroxide-gold modified electrode is the same as that employed in [12], where XPS measurements show the formation of a uniform nickel film. As SR responses of the bare gold electrode and that of the nickel hydroxide-gold modified electrode within the potential range $-0.3 \mathrm{~V}<E<0.35 \mathrm{~V}$ are the same, the presence of the nickel hydroxide layer in the $\mathrm{Au} / \mathrm{Ni}$ electrode does not add scattering centres to the conduction electrons at the gold film surface coated by $\mathrm{Au}(\mathrm{OH})_{\text {ads }}$ and $\mathrm{AuO}$ species. Thus, the nickel hydroxide layer does not directly interact with the gold film surface. This finding seems to be in agreement with previous XPS results [12], where it was not possible to confirm any direct interaction between gold and nickel oxide species. So, one cannot obtain structural information about the nickel hydroxide layer employing SR measurements within the potential range $-0.3 \mathrm{~V}<E<0.35 \mathrm{~V}$. However, considering that the surface deposition of nickel hydroxide films involves the participation of $\mathrm{OH}^{-}$ions, after the reduction of $\mathrm{AuO}$ and desorption of $\mathrm{Au}-\mathrm{OH}_{\mathrm{ads}}$ have just occurred, nickel adatom layers are formed and directly interact with the gold film surface increasing the $\mathrm{SR}$. Then, the $\Delta R$ increase within the potential range comprised between $-0.3 \mathrm{~V}$ and $-0.5 \mathrm{~V}$ can be attributed to the physical (non-faradaic) interaction of nickel adatom layers with the gold surface. Some structural information about the nickel adatom layers formed at potential values lower than $-0.3 \mathrm{~V}$ can be obtained from SR measurements. The $\Delta R$ increase with the increase in the nickel adatom surface coverage and its saturation, shown in Fig. 7, can be interpreted, from the microscopic point of view, in terms of the specularity parameter change ( $\Delta p=-k_{\mathrm{o}} \Gamma_{\text {Surf }}$ ) employing Eq. (3), and considering that the more dense the surface layer is, the more specularly the electrons are reflected [1]. The $\Delta p$ difference between the specularity parameter at $E=-0.5 \mathrm{~V}\left(p_{(E=-0,5 \mathrm{~V})}\right)$ and that at $E=-0.3 \mathrm{~V} p_{(E=-0.3 \mathrm{~V})}$ is a negative number, that is, $\Delta p=p_{(E=-0,5 \mathrm{~V})}-p_{(E=-0.3 \mathrm{~V})}<0$ because always $p_{(E=-0,5 \mathrm{~V})}<p_{(E=-0.3 \mathrm{~V})}$. That is, the electron reflection is more diffuse

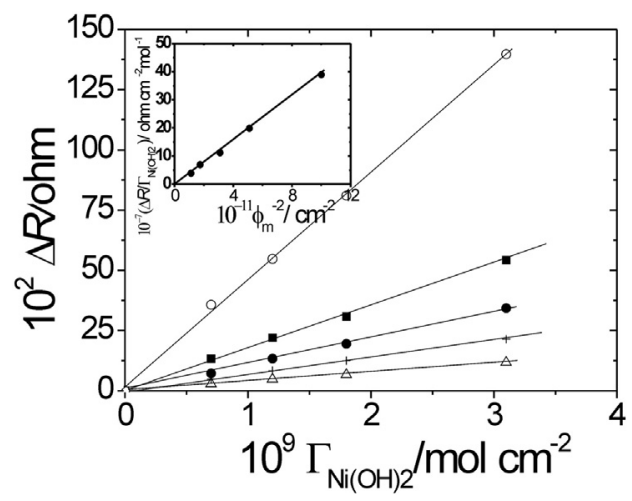

Fig. 8. $\Delta R$ vs. $\Gamma_{\mathrm{Ni}(\mathrm{OH}) 2}$ for different gold film thicknesses within the nickel hydroxide surface coverage range $0<\Gamma_{\mathrm{Ni}(\mathrm{OH}) 2}<3 \mathrm{nmol} \mathrm{cm}^{-2}$. Gold film thickness, $\varnothing_{\mathrm{m}}$ : (O) $10 \mathrm{~nm}$; (匹)14 nm; $(\bullet) 18 \mathrm{~nm} ;(+) 24 \mathrm{~nm} ;(\Delta) 30 \mathrm{~nm}$. Inset: $\Delta R / \Gamma_{\mathrm{Ni}(\mathrm{OH}) 2} \mathrm{vs} . \phi_{\mathrm{m}}^{-2}$. at $E=-0.5 \mathrm{~V}$ than at $E=-0.3 \mathrm{~V}$. Thus, as $\Gamma_{\mathrm{Ni}(\mathrm{OH}) 2}$ increases, $\Delta p$ increases in Eq. (3) because $p_{(E=-0,5 \mathrm{~V})}$ decreases while $p_{(E=-0.3 \mathrm{~V})}$ remains constant. Although absolute values of $\left.p_{(E}=-0,5 \mathrm{v}\right)$ and $\left.p_{(E}=-0.3 \mathrm{~V}\right)$ are unknown, the $\Delta R$ saturation is indicative of a $\left.p_{(E}=-0,5 \mathrm{~V}\right)$ limiting value at a given $\Gamma_{\mathrm{Ni}(\mathrm{OH}) 2}$ value. Then, in terms of the electron conduction scattering at a gold film surface coated with nickel adatoms, the initial linear resistance increase observed in Figs. 7 and 8 would mean that scatterer centres (nickel adatoms) for the gold conduction electrons are far apart from one another, forming part of a surface structure in which the nearest scatterer distances are constant and higher than the Fermi wavelength [13] of the conduction electrons of the substrate (gold). The latter would correspond to an island growth mechanism, as proposed by several authors for explaining SR data of different adsorbates on thin metal films [1]. As the nickel adatom surface coverage increases further, more compact structures that reflect electrons more specularly are formed, leading in these regions to no change or a decrease of SR. The appearance of these structures makes, on average, $\Delta R$ depart from the initial straight line, leading to a smaller slope and eventually to a constant value of $\Delta R$ (Fig. 7). This would be the point where areas occupied by surface structures reflecting diffusely are, on average, compensated by those reflecting specularly. If surface structures reflecting more specularly predominate over those reflecting diffusely, $\Delta R$ should decrease. However, the latter is not observed, at

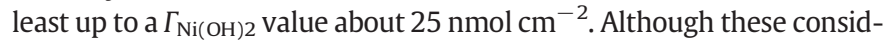
erations about the structure of the nickel films formed are only based on the free electron scattering model [13], XPS measurements also support the hypothesis that nickel film growth on the gold surface involves an initial stage in which nickel islands, with a random distribution, are formed [12].

With regard to the catalytic activity of the nickel hydroxide-gold modified electrode, it was demonstrated that the electrode combines the catalytic activity of gold species at low potentials $(E<0.3 \mathrm{~V})$ and that of $\mathrm{NiOOH}$ at higher polarisation potentials $(E<0.5 \mathrm{~V})[12,23]$. Besides, the good electronic and permeation properties of the nickel hydroxide film observed by CV, SR measurements within the potential range $-0.3 \mathrm{~V}<E<0.35 \mathrm{~V}$, show that the nickel hydroxide film does not affect the scattering processes of $\mathrm{Au}(\mathrm{OH})_{\text {ads }}$ species for the gold conduction electrons. Then, in agreement with previous work [12,23], it should be expected that even in the presence of the nickel film, the catalytic activity of the adsorbed hydroxide gold species at potential values lower than $-0.3 \mathrm{~V}<E<0.35 \mathrm{~V}$ will remain unaltered. Unfortunately, SR measurements cannot be extended to positive potential values beyond $E=0.35 \mathrm{~V}$, where $\mathrm{NiOOH}$ species predominate on the gold surface. However, nickel adatom deposition on the gold film substrate detected by SR measurements at potential values more negative than $-0.3 \mathrm{~V}$ may alter the reactivity of the base electrode and then, it can be used to change its electrocatalytic properties. The activity of the electrode depends on the formation of compact and uniform adsorbate layers on the substrate. In this regard, the saturation of the SR shown in Fig. 7 is indicative of a limiting coverage of the nickel adatom layer on gold. It is wellknown that just the deposition of a fraction or more than a monolayer of foreign atoms adsorbed on a base metal substrate electrode is often the first step in the metal electrodeposition and may affect the further growth of the deposit. In this regard, the so-called UPD refers to the formation of foreign metal adatoms at positive potentials of the reversible Nernst potential before its bulk deposition occurs [25]. This provides a unique method for controlling and changing the foreign metal adatom coverage under near thermodynamic equilibrium between zero and one monolayer. In the present study, the SR technique has proven to be adequate to study the deposition of a fraction of a Ni adatom monolayer on gold, even in the presence of a nickel hydroxide film on the gold surface. Since the adatom coverage is controlled only by the electrode potential, it is easy to investigate in situ electronic and structural properties of the deposit under well-defined conditions. The structure and morphology of Ni deposits, especially during the initial growth stages, have been extensively studied [26]. The electrochemistry of nickel has 
received much attention [27] and from the point of view of practical applications, nickel is widely used in the electroplating industry [28]. More work is in progress in our laboratory to obtain structural and electronic information about nickel adatom layers adsorbed on gold.

\section{Conclusions}

The electrochemical behaviours of a gold film electrode and a nickel hydroxide-gold film modified electrode in alkaline medium have been studied employing the surface resistance technique. With regard to the bare gold film electrode, the surface resistance response corresponding to hydroxide ion adsorption was analysed on the basis of the field effect theory. A relationship between the free electron charge density of the electrode in the presence and in the absence of adsorbed hydroxide ions has been obtained. This relationship indicates that in the presence of hydroxide ions, $77 \%$ of the electron density charge of gold is involved in hydroxide ion adsorption. Surface resistance data within the potential region corresponding to gold oxide formation were interpreted in terms of the size effect theory. A linear relation between the surface resistance change $(\Delta R)$ and the degree of oxidation $\left(\theta_{\mathrm{ox}}\right)$ at fixed gold film thickness $\left(\phi_{\mathrm{m}}\right)$ was obtained. Also, according to the size effect theory, the $\Delta R / \theta_{\mathrm{ox}}$ slope exhibits a linear dependence on $\phi_{\mathrm{m}}^{-2}$.

The comparison of SR responses of the nickel hydroxide-gold modified electrode with the bare gold film electrode within the potential range $-0.3 \mathrm{~V}<E<0.35 \mathrm{~V}$ shows that the nickel hydroxide layer does not affect the electron scattering process of $\mathrm{AuO}$ and $\mathrm{Au}-\mathrm{OH}_{\mathrm{ads}}$ layers formed on the gold surface. After the reduction of $\mathrm{OH}_{\text {ads }}$ and AuO layers, at potential values more negative than $-0.3 \mathrm{~V}$, nickel adatom layers are formed, which affects the scattering of gold conduction electrons. The electron conduction scattering model allows one to conclude that nickel film growth involves an initial stage in which nickel islands are formed, and then, compact structures (structures diffusely reflecting conduction electrons are, on average, compensated by those reflecting specularly) are formed. Despite the presence of the nickel film, the catalytic activity of the adsorbed hydroxide gold species at potential values lower than $-0.3 \mathrm{~V}<E<0.35 \mathrm{~V}$ remains unaltered. However, the deposition of a fraction or more than a monolayer of nickel adatoms on a base gold substrate electrode, at potential values lower than $-0.3 \mathrm{~V}$, may alter the reactivity of the base electrode and it can be used to change its electrocatalytic properties.

\section{Disclosure of potential conflicts of interest}

Conflict of interest: The author declares that he has not conflict of interest.

\section{Acknowledgements}

The author gratefully acknowledges the Consejo Nacional de Investigaciones Científicas y Técnicas (CONICET) (PIP No 0813/10) and also the Facultad de Ciencias Exactas, National University of La Plata (UNLP) (11/X693).

\section{References}

[1] J.W. Geus, in: J.R. Anderson (Ed.), Chemisorption and reactions on metallic films, Vol. 1, Academic Press, London 1971, pp. 365-368.
[2] R. Tucceri, A review about of the surface resistance technique in electrochemistry, Surf. Sci. Rep. 56 (2004) 85-157.

[3] R.I. Tucceri, Surface resistance measurements on thin gold film electrodes coated with poly(o-aminophenol) films, J. Electroanal. Chem. 505 (2001) 72-84

[4] R.I. Tucceri, Specularity change on a thin gold film surface coated with poly(oaminophenol) during the polymer redox conversion. The $\mathrm{pH}$ effect of the redox sites distribution at the metal/polymer interface, J. Electroanal. Chem. 543 (2003) 61-71.

[5] S. Madji, A. Jabbari, H. Heli, A study of the electrocatalytic oxidation of aspirin on a nickel hydroxide-modified nickel electrode, J. Solid State Electrochem. 11 (2007) 601-607.

[6] M. Jafarian, M.G. Mahjani, H. Heli, F. Gobal, M. Heydarpoor, Electrocatalytic oxidation of methane at nickel hydroxide modified nickel electrode in alkaline solution Electrochem, Communications 5 (2003) 184-188.

[7] M. Hajjizadeh, A. Jabbari, H. Heli, A.A. Mosavi-Movahedi, A. Shaffiee, K. Karimian, Electrocatalytic oxidation and determination of deferasirox and deferiprone on a nickel oxyhydroxide-modified electrode, Anal. Biochem. 373 (2008) 337-348.

[8] S. Berchmans, H. Gomathi, G.P. Rao, Electrooxidation of alcohols and sugars catalysed on a nickel oxide modified glassy carbon electrode, J. Electroanal. Chem. 394 (1995) 267-270.

[9] R.I. Tucceri, D. Posadas, Resistive behaviour of thin gold film electrodes under direct current polarization, J. Electrochem. Soc. 130 (1983) 104-107.

[10] R.I. Tucceri, D. Posadas, Theoretical approach to the resistive behaviour of thin solid film electrodes under direct current polarization, J. Electrochem. Soc. 128 (1981) 1478-1483.

[11] F.M. Romeo, R.I. Tucceri, D. Posadas, Surface conductivity changes during the electrochemical adsorption of UPD layers on silver and gold, Surf. Sci. 203 (1988) $186-200$.

[12] I.G. Casella, M.R. Guascito, M.G. Sannazzaro, Voltammetric and XPS investigations of nickel hydroxide electrochemically dispersed on gold surface electrodes, J. Electroanal. Chem. 462 (1999) 202-210.

[13] J.M. Ziman, The Principles of the Theory of Solids, second ed. Cambridge University Press, London, 1972.

[14] F. Fuchs, The conductivity of thin metallic films according to the electron theory of metals, Proc. Camb., Phyl. Soc. Math. Phys. Sci. 34 (1938) 100.

[15] E.H. Sondheimer, The mean free path of electrons in metals, Adv. Phys. 1 (1952) 1.

[16] A. Chen, J. Lipkowski, Electrochemical and spectroscopic studies of hydroxide adsorption at Au (111) electrode, J. Phys. Chem. B 103 (1999) 682-691.

[17] D.W. Kirk, F.R. Foulkes, W.F. Graydon, The electrochemical formation of $\mathrm{Au}(\mathrm{I})$ hydroxide on gold in aqueous potassium hydroxide, J. Electrochem. Soc. 127 (1980) 1069-1076.

[18] J. DeDilvestro, M.J. Weaver, Surface structural changes during oxidation of gold electrodes in aqueous media as detected using surface-enhanced Raman spectroscopy, J. Electrocardiol. 209 (1986) 377-386.

[19] W.J. Anderson, W.N. Hansen, Observing the electrochemical interface vis electrode surface conductance, J. Electroanal. Chem. 43 (1973) 329-338.

[20] J. Taraszewska, G. Roslonek, Electrocatalytic oxidation of methanol on a glassy carbon electrode modified by nickel hydroxide formed by ex-situ chemical precipitation, J. Electroanal. Chem. 364 (1994) 209-213.

[21] J. Joseph, H. Gomathi, G.P. Rao, Electrochemical characteristics of thin films of nickel hexacyanoferrate formed on carbon substrates, Electrochim. Acta 36 (10) (1991) $1537-1541$

[22] M. Fleishmann, K. Korinek, D. Pletcher, The oxidation of organic compounds at a nickel anode in alkaline solution, J. Electroanal. Chem. 31 (1971) 39-49.

[23] I.G. Casella, M.R. Guascito, T.R.I. Cataldi, Electrocatalysis and amperometric detection of alditols and sugars at a gold-nickel composite electrode in anion-exchange chromatography, Anal. Chim. Acta 398 (1999) 153-160.

[24] R. Tucceri, D. Posadas, Surface structural changes of evaporated thin silver films electrodes during the adsorption of chloride ion, J. Electroanal. Chem. 270 (1989) 415-419.

[25] A. Aramata, in: J.-O.'.M. Bockris, R.E. White, B.E. Conway (Eds.), Modern Aspects in Electrochemistry, vol. 31, Plenum Press 1997, p. 181.

[26] E.A. Moller, J. Kintrup, A. Lachenwitzer, O.M. Magnussen, R.J. Bhem, In situ STM study of the electrodeposition and anodic dissolution of ultrathin epitaxial Ni films on $\mathrm{Au}(111)$, Phys. Rev. B 56 (1997) 12506.

[27] A.J. Arvía, D. Posadas, in: A.J. Bard (Ed.), Encyclopedia of the Electrochemistry of the Elements, Dekker, New York, 1975 Ch. 3.

[28] Z. Galus, L.J. Jeftic, Investigation of the kinetics and mechanism of the electroreduction of nickel(II) in thiocyanate and thiocyanato-pyridine media, J. Electroanal. Chem. 14 (1967) 415. 